\title{
Uso do manguezal de Guaratiba, Baía de Sepetiba, Rio de Janeiro, pelo peixe- rei Atherinella brasiliensis (Quoy \& Gaimard) (Atheriniformes, Atherinopsidae)
}

\author{
Leonardo M. Neves '; Hamilton H. Pereira 1; Marcus R. da Costa ${ }^{2} \&$ Francisco G. Araújo ${ }^{1}$ \\ ${ }^{1}$ Laboratório de Ecologia de Peixes, Universidade Federal Rural do Rio de Janeiro. Antiga Rodovia Rio-São Paulo, km 47, \\ 23851-970 Seropédica, Rio de Janeiro,Brasil.E-mail: leonardomneves@yahoo.com.br; gerson@ufrrj.br \\ ${ }^{2}$ Centro Universitário Módulo. Avenida Frei Pacífico Wagner 653, Centro, 11660-903 Caraguatatuba, São Paulo, Brasil.
}

\begin{abstract}
The use of the Guaratiba magrove, Sepetiba Bay, Rio de Janeiro, by the silverside Atherinella brasiliensis (Quoy \& Gaimard) (Atheriniformes, Atherinopsidae). Fish and environmental variables were sampled in a mangrove area from Sepetiba bay to assess the role of this system in life cycle of the silverside Atherinella brasiliensis (Quoy \& Gaimard, 1824). Standardized monthly sampling using beach seines were conducted at five sites, between August, 2002 and July, 2003 in daily (day/night) basis, with environmental variables being taken at each site. The highest numerical abundance was recorded in the intermediate sample area in the mangrove channel and the lowest in the innermost site. The highest weight occurred in the outermost site near to the sea connection. Numerical abundance was directly correlated with temperature and salinity, and weight with salinity and dissolved oxygen $(p<0.05)$. Size ranged from 10 to $160 \mathrm{~mm} \mathrm{TL}$, with modes of $40-50 \mathrm{~mm}$ TL occurring all over the year in all sites. Recruitment occurred all year round but peaks $(T L=30 \mathrm{~mm})$ in October-December, 2002 and July, 2003 were recorded afterwards the records of larger fishes (September, 2002 and June, 2003) mainly in the outermost site. The largest fishes concentrated in the sandy substrate of the outermost sites, areas more suitable for $A$. brasiliensis spawning, with recruits dispersing all over the mangrove areas, which are used as rearing grounds. The affinity for highly structured habitats in mangrove and the capacity to use efficiently semiclosed systems was confirmed for A. brasiliensis in Guaratiba mangrove.
\end{abstract}

KEY WORDS. Coastal fishes; distribution; size; mangrove.

RESUMO. Coletas padronizadas de peixes e tomadas de variáveis ambientais foram realizadas em uma região de mangue na Baía de Sepetiba visando avaliar o papel deste sistema no ciclo de vida do peixe-rei Atherinella brasiliensis (Quoy \& Gaimard, 1824). Um programa de amostragens mensais de arrastos de praia foi realizado, em cinco locais em uma área de mangue, entre agosto de 2002 e julho de 2003 nos períodos diurno e noturno, com variáveis ambientais tendo sido tomadas em cada local. As maiores abundâncias numéricas foram registradas no local da zona intermediária do canal principal, e as menores no local mais interno. As maiores contribuições em peso ocorreram no local mais externo e próximo da conexão com o mar. A abundância numérica foi diretamente correlacionada com a temperatura e salinidade, e o peso, com a salinidade e oxigênio dissolvido ( $p$ $<0,05)$. A população amostrada variou de 10 a $160 \mathrm{~mm} \mathrm{CT}$, com moda de $40-50 \mathrm{~mm}$ CT durante todo o ano em todos os locais. O recrutamento ocorre durante todo o ano, com picos $(C T=30 \mathrm{~mm})$ de outubro a dezembro de 2002 e julho de 2003, tendo sido registrados após a ocorrência dos peixes de maiores tamanhos em setembro de 2002 e junho de 2003 principalmente no local mais externo. Os maiores indivíduos se concentraram no local mais externo de substrato arenoso, áreas mais propícias para reprodução de $A$. brasiliensis, com os recrutas dispersando-se ao longo de todos os locais do canal do mangue que são utilizados como área de criação. A afinidade por habitats bem estruturados e a capacidade de usar eficientemente sistemas semifechados foi confirmada para $A$. brasiliensis no manguezal de Guaratiba.

PALAVRAS-CHAVE. Distribuição; peixes costeiros; manguezal; tamanho.

O peixe-rei Atherinella brasiliensis (Quoy \& Gaimard, 1824) distribui-se da Venezuela ao Rio Grande do Sul onde habita regiões costeiras principalmente na desembocadura de rios e em águas salobras, sendo considerada uma espécie estuarina residente em alguns ecossistemas (ANDREATA et al. 1990, Araújo et al. 1997, Hostim-Silva et al. 1995). Alimenta-se principalmente de invertebrados bentônicos, insetos dípteros e crustáceos planctônicos (Bemvenuti 1990).

Revista Brasileira de Zoologia 23 (2): 421-428, junho 2006 
Esta espécie tem sido registrada entre as mais abundantes em lagoas costeiras do Rio de Janeiro, como a laguna do Marapendi (ANDreata et al. 1990), a laguna de Jacarepaguá (Andreata et al. 1992) e a laguna Rodrigo de Freitas (Andreata et al. 1997). Na Baía de Sepetiba (22 $53^{\prime}-23^{\circ} 04^{\prime} S$; 043³3'$044^{\circ} 05^{\prime} \mathrm{W}$ ), uma área semi-aberta de aproximadamente $520 \mathrm{~km}^{2}$ no Sudeste do Estado Rio de Janeiro, esta espécie tem sido reportada em cerca de $31,25 \%$ das amostragens de arrasto de praias, correspondendo a 1,8\% do número total e 5,63\% do peso em arrastos experimentais de fundo (Pessanha \& Araújo 2003). Na parte mais protegida, localizada no extremo leste da baía, encontra-se a Reserva Biológica e Arqueológica de Guaratiba, uma extensa área de manguezal com 2800 ha, constituindo a principal área da baía em produtividade biológica (SEMA 1998). Manguezais são regiões costeiras de grande importância no ciclo de vida de espécies de peixes em sistemas costeiros semifechados por servirem de áreas de desova, berçários, proteção contra predadores e áreas de alimentação. Estes ambientes apresentam em comum com as lagoas costeiras, uma maior homogeneidade das condicionantes ambientais, onde os peixes poderiam tirar proveito da energia e elevada produtividade dos mesmos (LAEDSGAARD \& JoHnson 2001), além de evitar a competição com espécies marinhas nas praias arenosas (GIANNINI \& Paiva-Filho 1995, Gaelzer et al. 2004). Adicionalmente, os habitats não permanecem constantes, variando regularmente em bases diárias e sazonais. Espécies que habitam as zonas rasas utilizam numerosas estratégias, principalmente associadas com a alimentação, tolerância às condições físico-químicas adversas e fuga de predadores, levando a complexos movimentos sazonais e diuturnais (BurRows et al. 1994).

O canal de mangue, objeto do presente estudo, tem uma extensão de aproximadamente de $2 \mathrm{~km}$, com densas formações de mangue vermelho (Rhyzophora mangle), onde o movimento das marés constitui um importante fator estruturador do sistema, tornando expostas tais praias durante a baixa maré. O substrato tende a ser mais arenoso nas zonas próximas da conexão com o mar e lodoso, nas zonas mais internas.

O presente trabalho visa estudar os padrões de utilização do manguezal de Guaratiba por A. brasiliensis, com enfoque na estrutura de tamanho, na distribuição espacial e temporal (sazonal e diuturnal), bem como as relações com as variáveis ambientais. Procurou-se comparar os padrões encontrados no manguezal de Guaratiba com outros ambientes de diferentes níveis de estruturação e conexão com o mar, como lagoas costeiras (semi-fechados) e baías (semi-abertos).

\section{MATERIAL E MÉTODOS}

Amostragens mensais de arrastos de praia foram realizadas entre agosto de 2002 e julho de 2003 em dois períodos: diurno e noturno. Os arrastos foram padronizados sendo realizado perpendicular à margem da praia, a uma profundidade inferior a 1,5 m, com extensão de aproximadamente $30 \mathrm{~m}$. Foram amostradas cinco praias, formadas na região do manguezal, ao longo dos canais de marés na baixa-mar, em um gradiente mar-interior do manguezal: 1$)$ Local $1\left(23^{\circ} 02,730^{\prime} \mathrm{S}-\right.$ $\left.043^{\circ} 33,540^{\prime} \mathrm{W}\right)$, situado mais no interior no manguezal, distanciando-se aproximadamente $2 \mathrm{~km}$ da conexão com o mar, com características mais peculiares de manguezal, grande quantidade de matéria orgânica e substrato lodoso; 2) Local 2, é a praia de menor profundidade, sendo um pequeno canal adjacente ao canal principal; 3) Local 3 é o canal mais profundo, situado no canal principal; 4) Local 4 apresenta maior influência marinha, em comparação com os locais citados anteriormente, com substrato areno-lodoso; e 5) Local 5 (2303,566's; $043^{\circ} 34,056^{\prime} \mathrm{W}$ ) é o mais externo, sendo pouco profundo e situado junto da comunicação com o mar, com substrato arenoso.

As coletas de peixes foram feitas com uma rede de arrasto de praia (picaré) com $10 \mathrm{~m}$ x 2,5 m; malha de $7 \mathrm{~mm}$ entre nós consecutivos. Cada arrasto cobriu uma extensão de aproximadamente $30 \mathrm{~m}$ por $7 \mathrm{~m}$ de largura. A unidade amostral foi padronizada como o total de peixes capturados por arrasto, tendo sido feitas tréplicas das amostras em cada local. A cada arrasto foram tomados as variáveis ambientais de temperatura, salinidade, oxigênio dissolvido e condutividade, com um multisensor YSI 85.

A abundância relativa, CPUE, foi avaliada através do número e peso dos peixes por arrasto. Comparações espaciais (locais de coleta) e temporais (estações do ano e dia/noite) das CPUEs e das variáveis ambientais foram feitas com análise de variância, seguida, a posteriori, do teste de Tukey $(\mathrm{p}<0,05)$, com os dados brutos tendo sido previamente centrados e reduzidos, para atender os requisitos de normalidade e homocedasticidade desta técnica paramétrica (ZAR 1999). Para o critério de sazonalidade, os meses de amostragem foram agrupados da seguinte forma: Inverno/primavera (agosto, setembro e outubro), primavera/verão (novembro, dezembro e janeiro), verão/outono (fevereiro, março, abril) e outono/inverno (maio, junho, julho). Para determinar relações entre as variáveis ambientais com as CPUEs (número e peso) foram utilizadas a correlação de postos de Spearman e a análise de regressão múltipla (forward stepwise).

O crescimento foi estudado através das distribuições de freqüências de comprimento, com os indivíduos agrupados em classes de $5 \mathrm{~mm}$ de comprimento total (CT). As freqüências mensais foram analisadas utilizando a subrotina ELEFAN I do programa FISAT (Gayanilo-Jr. \& Pauly 1997), que também serviu para determinação de $\mathrm{K}$ (coeficiente de crescimento) e $\phi$ (índice de performance). O parâmetro L $\infty$ (comprimento assintótico) foi determinado pela equação de Taylor ( $\infty \propto=$ Lmáx/0,95).

\section{RESULTADOS}

\section{Parâmetros ambientais}

Temperatura: as temperaturas médias apresentaram diferenças altamente significativas ( $p<0,01$, ANOVA) entre as estações do ano (Tab. I), sendo registradas as maiores médias $\left(26,98^{\circ} \mathrm{C}\right)$ no verão/outono e as menores $\left(23,52^{\circ} \mathrm{C}\right)$ no inverno/primavera. Não foram detectadas significativas diferenças entre os locais de amostragem, nem entre o dia e a noite. 
Tabela I. Valores de F (ANOVA) e diferenças significativas das comparações das CPUE - número e CPUE - peso e fatores ambientais de Atherinella brasiliensis nos 12 meses de amostragem no manguezal de Guaratiba, Baía de Sepetiba, Rio de Janeiro.

\begin{tabular}{lcccccccc}
\hline \multicolumn{1}{c}{ Fatores } & Local & Fotoperíodo & Estação & LxF & LxE & FxE & LxFxE & Tukey \\
\hline Número & $15,77^{* *}$ & NS & $7,23^{* *}$ & NS & NS & $4,03^{* *}$ & $3,21^{* *}$ & $3>4,2,5>1 \mathrm{~V} / \mathrm{O}, \mathrm{P} / \mathrm{V}>\mathrm{O} / \mathrm{I}, \mathrm{I} / \mathrm{P}$ \\
Peso & $28,39^{* *}$ & $\mathrm{NS}$ & $\mathrm{NS}$ & $2,92^{*}$ & $\mathrm{NS}$ & $5,08^{* *}$ & $3,13^{* *}$ & $5>3,4>2,1$ \\
Temperatura & $2,42^{*}$ & $\mathrm{NS}$ & $51,40^{* *}$ & $5,96^{* *}$ & $2,53^{* *}$ & $\mathrm{NS}$ & $\mathrm{NS}$ & $\mathrm{V} / \mathrm{O}>\mathrm{P} / \mathrm{V}>\mathrm{O} / \mathrm{I}, \mathrm{I} / \mathrm{P}$ \\
Salinidade & $15,33^{* *}$ & $\mathrm{NS}$ & $17,63^{* *}$ & $3,56^{* *}$ & $4,60^{* *}$ & $3,45^{*}$ & $7,18^{* *}$ & $2,3,4>1,5 \mathrm{I} / \mathrm{P}, \mathrm{O} / \mathrm{I}>\mathrm{P} / \mathrm{V}, \mathrm{V} / \mathrm{O}$ \\
$\begin{array}{l}\text { Oxigênio } \\
\text { dissolvido }\end{array}$ & $14,50^{* *}$ & $30,65^{* *}$ & $21,29^{* *}$ & $10,53^{* *}$ & $2,79^{*}$ & $4,34^{*}$ & $\mathrm{NS}$ & $2,3,4,5>1 \mathrm{I} / \mathrm{P}, \mathrm{P} / \mathrm{V}>\mathrm{V} / \mathrm{O}, \mathrm{O} / \mathrm{I} \mathrm{dia}>\mathrm{noite}$ \\
Condutividade & $7,59^{* *}$ & $\mathrm{NS}$ & $9,10^{* *}$ & $5,01^{* *}$ & $\mathrm{NS}$ & $3,90^{* *}$ & $4,78^{* *}$ & $2,3,4>1,5 \mathrm{I} / \mathrm{P}, \mathrm{V} / \mathrm{O}, \mathrm{O} / \mathrm{I}>\mathrm{P} / \mathrm{V}$ \\
\hline
\end{tabular}

$\left.\left.{ }^{*}\right) \mathrm{p}<0,05,{ }^{* *}\right) \mathrm{p}<0,01-\mathrm{I} / \mathrm{P}$ (Inverno/primavera); P/V (Primavera/verão); V/O (Verão/outono); O/I (Outono/inverno). Locais de amostragem: 1, 2, 3, 4 e 5 .

Salinidade: as salinidades médias apresentaram diferenças significativas $(\mathrm{p}<0,01)$, entre as estações do ano, apresentando maiores valores médios $(33,5)$ no inverno/primavera e menores valores $(32,01)$ no verão/outono (Tab. I). Foram encontradas diferenças significativas entre os locais de amostragem, com as maiores médias $(33,58)$ no local 3 , e as menores $(31,80)$ no local 1. Não foram registradas diferenças significativas no período dia/noite (Tab. I).

Oxigênio dissolvido: foram encontradas diferenças significativas $(\mathrm{p}<0,01)$ para oxigênio dissolvido entre as estações do ano, com os maiores valores médios $(82,18 \%)$ registrados no inverno/primavera e menores $(68,48 \%)$ no verão/outono (Tab. I). Diferenças altamente significativas foram detectadas entre os locais de amostragem e o fotoperíodo, com os maiores valores no local 3 (77,55\%), e os menores no local 1 (62,94\%); também durante o dia $(80,16 \%)$ foram registrados os maiores valores em relação ao período da noite $(71,66 \%)$ (Tab. I).

Condutividade: diferenças significativas $(p<0,01)$ foram detectadas para condutividade entre as estações do ano, apresentando os maiores valores médios $(50,86 \mathrm{mS} / \mathrm{cm})$ no inverno/primavera e menores $(48,59 \mathrm{mS} / \mathrm{cm})$ na primavera/verão (Tab. I). Dentre os locais de amostragem, os maiores valores médios foram registrados nos locais 2,3 e $4(50,68 \mathrm{mS} / \mathrm{cm})$, enquanto os menores no local $1(40,65 \mathrm{mS} / \mathrm{cm})$ e no local 5 $(49,28 \mathrm{mS} / \mathrm{cm})$. Ao longo do fotoperíodo não foram registradas diferenças significativas (Tab. I).

\section{Distribuição espacial e temporal}

Um total de 21.425 indivíduos de $A$. brasiliensis foi capturado, pesando $25.443,78 \mathrm{~g}$, nas 360 amostras nos arrastos de praia. Estes valores corresponderam a $57,92 \%$ do número total de todas as espécies de peixes capturadas, e a $54,82 \%$ do peso total, com uma freqüência de ocorrência de 93,61\% das amostras. Uma ampla distribuição desta espécie foi registrada ao longo de todos os locais de amostragem, em todas as estações do ano e durante os períodos diurno e noturno. Diferenças significativas para as CPUEs (número e peso) foram encontradas entre os locais de coletas e as estações do ano (Tab. I e Fig.1).

Espacialmente as maiores CPUEs - número foram regis- trados no local 3, enquanto os menores valores ocorreram no local 1. Para CPUEs - peso os maiores valores ocorreram no local 5 e os menores no local 1.

Sazonalmente, as maiores CPUEs - número ocorreram durante a primavera/verão, verão/outono, e os menores no outono/inverno e inverno/primavera. As CPUEs - peso não apresentaram diferenças significativas entre as estações do ano.

O fotoperíodo não apresentou diferenças significativas tanto para as CPUEs - número, como para as CPUEs - peso.

\section{Influência das variáveis ambientais}

A análise de correlação de postos de Spearman apresentou associações positivas significativas da temperatura $\left(0,129^{*}\right)$ e salinidade $\left(0,115^{*}\right)$ com o número de indivíduos, e da salinidade $\left(0,157^{* *}\right)$ e o oxigênio dissolvido $\left(0,189^{* *}\right)$ com o peso. A análise de regressão múltipla apresentou significativos coeficientes de regressão somente para a salinidade com o número $\left(0,217^{\star}\right)$ e com o peso $\left(0,133^{*}\right)$. As demais variáveis independentes não foram incluídas no modelo.

\section{Estrutura de tamanho}

A população de $A$. brasiliensis no manguezal de Guaratiba apresentou indivíduos com tamanho variando entre 10 a 160 $\mathrm{mm}$ CT. Peixes de maior tamanho (CT $>100 \mathrm{~mm}$ ) ocorreram nos meses de setembro de 2002 e junho de 2003 (Fig. 2); por outro lado, indivíduos de menor tamanho $(\mathrm{CT}=30 \mathrm{~mm} \mathrm{CT})$ ocorreram principalmente nos meses de outubro a dezembro de 2002, e julho de 2003. No entanto, uma moda de 40-50 mm CT foi observada durante todos os meses do ano (Fig. 2). Através do acompanhamento da progressão modal, ao final do primeiro ano $A$. brasiliensis atingiu cerca de $90 \mathrm{~mm}$ CT. Três coortes ou grupos de idade foram obtidos do programa FISAT, embora a de maior tamanho tenha sido pouco representada nas amostragens. Com base nas distribuições de freqüências de comprimento foram calculados os valores de $\mathrm{K}=0,9$ e $\phi=4,41$ através da subrotina ELEFAN I. O tamanho máximo assintótico $(\mathrm{L} \infty)$ determinado de acordo com a equação de Taylor foi de CT $=168,4 \mathrm{~mm}$.

A estrutura de tamanho não variou entre os períodos dia/ noite (Fig. 3), com os indivíduos de CT = 40-50 mm sendo mais abundantes em todos os locais. Peixes de maior tamanho 


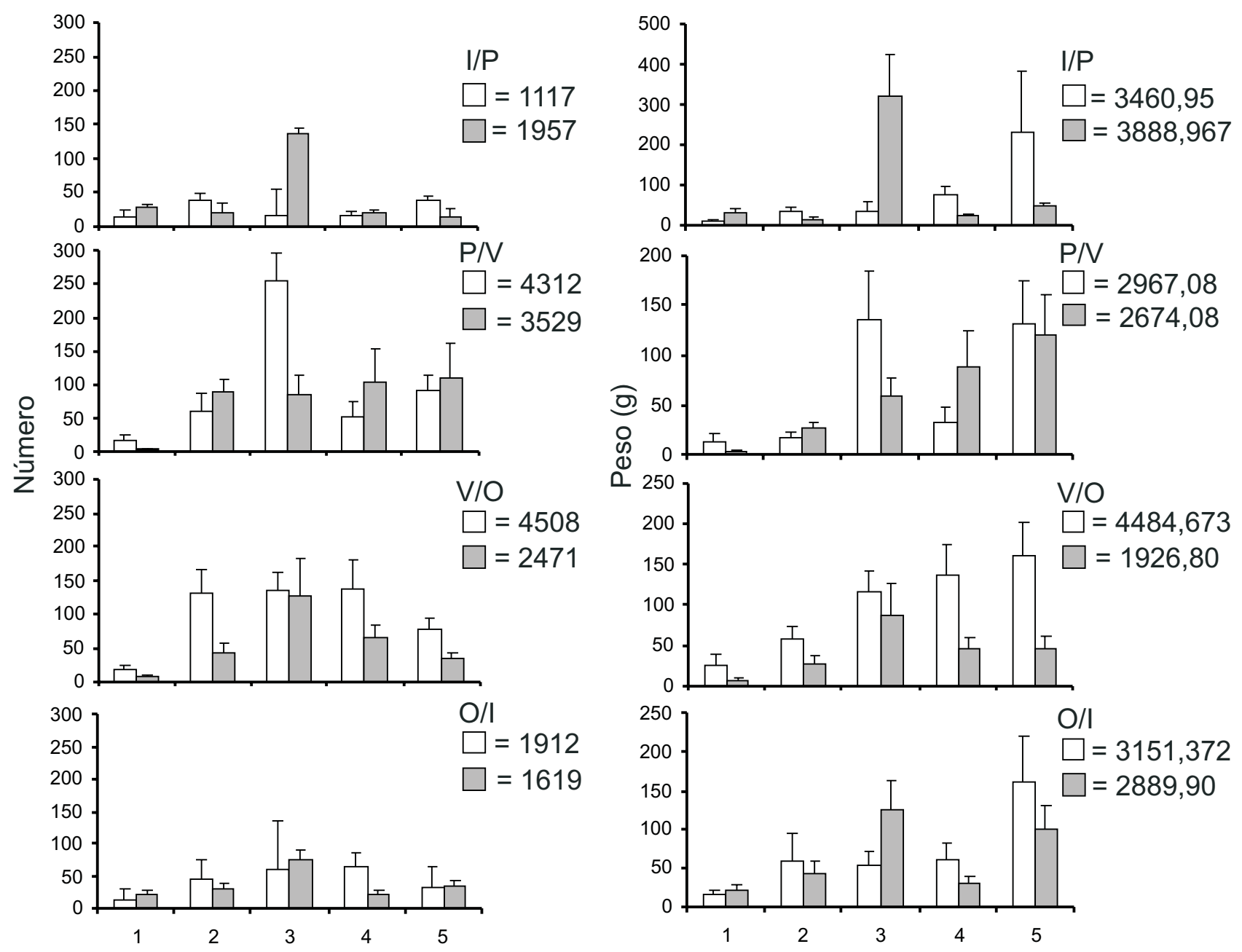

Figura 1. CPUE número e peso (g) de A. brasiliensis no manguezal de Guaratiba, Rio de Janeiro, entre agosto de 2002 e julho de 2003. Estações do ano: (I/P) inverno/primavera, (P/V) primavera/verão, (V/O) verão/outono, (O/I) outono/inverno; locais de amostragem: 1, 2, 3, 4 e 5 ; (d) dia, (n) noite.

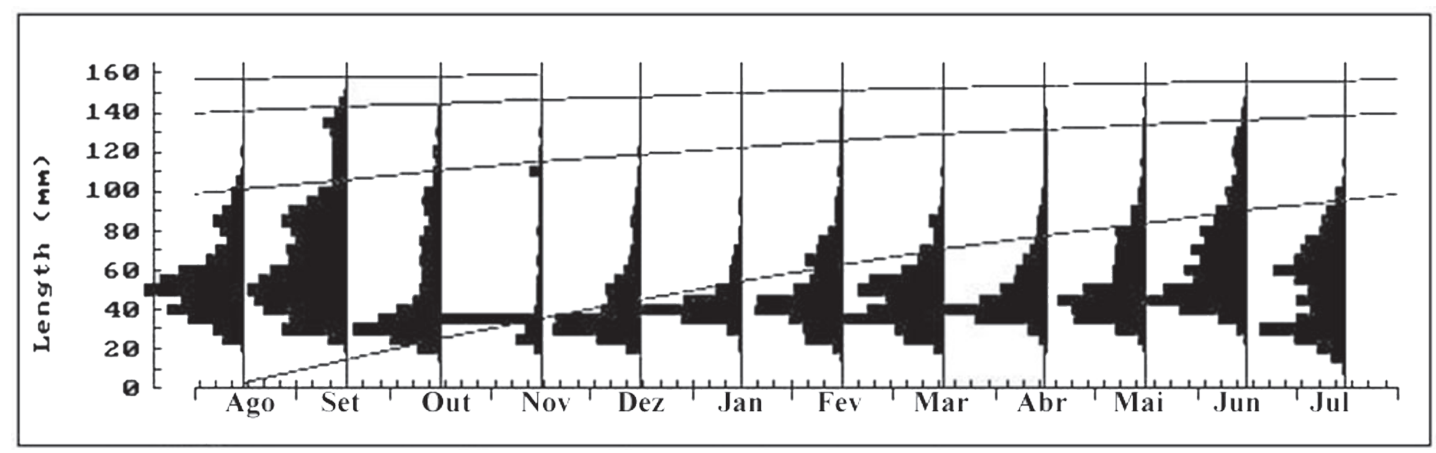

Figura 2. Progressão modal mensal com as respectivas coortes, obtidas através da subrotina ELEFAN I do programa FISAT. Número de indivíduos: $\mathrm{Ago}=1409, \mathrm{Set}=1077, \mathrm{Out}=592, \mathrm{Nov}=916, \mathrm{Dez}=4459, \mathrm{Jan}=890, \mathrm{Fev}=1851, \mathrm{Mar}=1217, \mathrm{Abr}=1526, \mathrm{Mai}=1218$, Jun $=1179$, Jul = 778 .

Revista Brasileira de Zoologia 23 (2): 421-428, junho 2006 


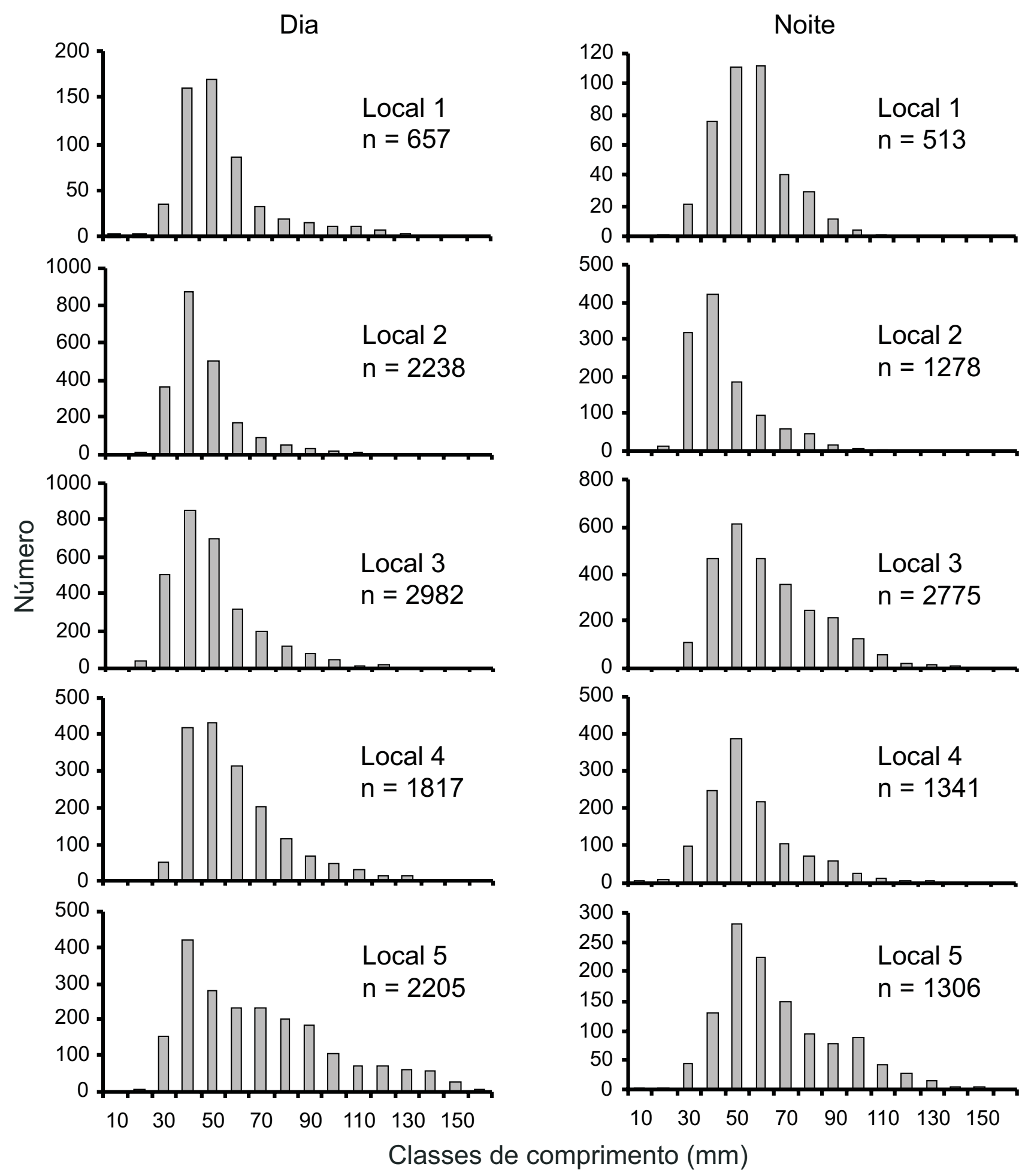

Figura 3. Distribuição de freqüências de comprimento por local de amostragem e fotoperíodo de $A$. brasiliensis no manguezal de Guaratiba, RJ, entre agosto de 2002 e julho de 2003. Locais de amostragem: 1, 2, 3, 4 e 5. 
(CT > $100 \mathrm{~mm}$ ) ocorreram principalmente no local 5, mais próximo da comunicação com o mar e em menor instância, no local 4.

\section{DISCUSSÃO}

Atherinella brasiliensis apresentou-se como uma espécie residente e dominante no manguezal de Guaratiba, e provavelmente é adaptada para explorar ambientes costeiros tropicais protegidos de elevada complexidade estrutural e marcada dinâmica das marés. Suas elevadas ocorrências em ampla distribuição de tamanhos, encontradas ao longo de todo o ano e nos diferentes períodos (sazonal e dia/noite) e locais estudados, são indicações da adequabilidade do ambiente para uso por esta espécie. A participação desta espécie em arrastos de praias ao longo da margem continental da Baía de Sepetiba tem sido registrada com contribuições relativas bem inferiores aos do manguezal de Guaratiba. Pessanha \& ARAúJo (2003) registraram uma abundância relativa de somente $1,8 \%$ do número e 5,63\% do peso em arrastos de praia na Baía de Sepetiba no período de agosto de 1998 a junho de 1999, o que contrasta em muito com o observado neste trabalho $(57,92 \%$ do número e $54,82 \%$ do peso). Adicionalmente, as maiores abundâncias relativas encontradas por Pessanha \& Araújo (2003) ocorreram nas praias de Itacuruçá e Coroa Grande, que se situam nas proximidades de uma segunda formação de manguezais da Baía de Sepetiba; estes maiores valores relativos indicam uma contribuição/exportação de $A$. brasiliensis para estas praias a partir da área de manguezais.

Um decréscimo nas contribuições relativas de $A$. brasiliensis para as assembléias de peixes de praias arenosas da Baía de Sepetiba parece estar ocorrendo ao longo dos últimos anos, um provável efeito da redução do manguezal de Coroa Grande/ Itacuruçá, como resultado da expansão desordenada de ocupação da área. Informações históricas na Baía de Sepetiba indicam esta tendência. Nos arrastos realizados entre julho de 1983 a junho de 1984 A. brasiliensis destacou-se entre os mais abundantes grupos de peixes, ocupando o quarto lugar em abundância numérica, contribuindo com $10 \%$ do número total de peixes e estando presente em 52,5\% das amostras (PESSANHA et al. 2000); uma década depois (julho de 1993 a junho de 1994), esta espécie ocupou o quinto lugar, contribuindo com $6,7 \%$ do total de peixes, estando presente em 43,3\% das amostras (ARAújo et al. 1997). Os registros mais recentes (Pessanha \& Araújo 2003) indicam abundâncias relativas ainda mais reduzidas.

A distribuição de $A$. brasiliensis tem sido documentada em lagoas costeiras do estado do Rio de Janeiro, como na laguna do Marapendi, onde participou com 41,7\% do número total de peixes (ANDreata et al. 1990) e na lagoa Rodrigo de Freitas, com participação relativa de 31,41\% (ANDREATA et al. 1997). Na região da Laguna, Santa Catarina, a contribuição numérica de $A$. brasiliensis foi de 11,6\% (Monteiro-Neto et al. 1990). Estas elevadas contribuições relativas indicam que o ambiente semi-fechado lagunar é propício para ocorrência desta espécie, e que áreas mais abertas, como planícies de marés (4,9\% do número; 5,0\% do peso) da Baía de Paranaguá (Oliveira-Neto et al. 2004), praias adjacentes ao estuário do rio Itabapoana ( $<2,0 \%$ número) (GoMEs et al. 2003) são menos favoráveis para o seu desenvolvimento. Em rios de marés (gamboa do Sucuriú) da Baía de Paranaguá, uma área meandrica de alto dinamismo e vegetação composta por marismas e manguezais, $A$. brasiliensis foi uma das espécies mais abundantes, contribuindo com 7,8\% da captura total (SPACH et al. 2003). Portanto, é razoável hipotetizar que a existência de sistemas estruturados e semi-fechados, como os manguezais, são ambientes favoráveis, enquanto as praias arenosas, especialmente aquelas pouco protegidas e com baixo níveis de estruturação apresentam limitações na distribuição desta espécie.

O uso de regiões de mangue pode estar associado à disponibilidade de recursos alimentares e diversidade de microhabitats característicos deste tipo de ambiente. BLABER \& Blaber (1980) afirmam que ambientes com águas mais calmas e rasas, de pouca profundidade e vegetação abundante, constituem elevada produtividade, sendo consideradas ideais à criação de peixes juvenis por fornecerem proteção e alimento. LAEGDSGAARD \& JOHNSON (2001) simulando em laboratório a estrutura de manguezais utilizando raízes e pneumatóforos artificiais, constataram que estes, quando permitem o crescimento de algas, acarretam o acúmulo de pequenos invertebrados, atraindo até quatro vezes mais peixes juvenis do que em ambientes não estruturados, sendo consideradas então, importantes áreas para alimentação de muitas espécies de peixes.

Embora os locais amostrados sejam relativamente próximos, uma maior abundância numérica foi observada no local 3 , situado na região intermediária do canal principal; a maior profundidade deste local, provavelmente possibilita as maiores concentrações de peixes em relação aos demais locais. Os maiores pesos médios ocorreram no local 5, mais externo e junto com a conexão com o mar, e os menores no local 1, mais interno. Este gradiente sugere que os adultos (CT $>100 \mathrm{~mm})$ se concentram na parte mais externa, onde o substrato arenoso constitui ambiente mais favorável para reprodução. Embora não existam informações disponíveis sobre locais de desova de A. brasiliensis, é esperado que a mesma ocorra em praias arenosas. De acordo com Middaugh et al. (1981) e Middaugh \& Hemmer (1992) o atherinídeo Menidia menidia (Linnaeus, 1766) desova em zonas superiores da região entre-marés, fazendo o depósito dos ovos sobre a vegetação ou detritos acumulados nas praias arenosas, com o maior sucesso da desova coincidindo com as maiores marés. No caso do manguezal de Guaratiba, é portanto razoável supor que os ovos e larvas decorrentes de desovas no local 5, seriam carreados pelas marés, com os recrutas dispersando-se ao longo dos canais de mangue, onde se desenvolveriam, tirando proveito da maior estruturação, abrigos e águas pouco profundas. Uma provável indicação, é a ocorrência de recrutas de menor tamanho $(\mathrm{CT}=30 \mathrm{~mm})$ que apresentaram maiores abundâncias em outubro a dezembro de 2002, e julho de 2003, meses subseqüentes aos picos de indivíduos maiores (CT $>100 \mathrm{~mm}$ ) que ocorrem basicamente no local 5 em setem- 
bro de 2002 e junho de 2003. De acordo com Favaro et al. (2003) indivíduos de tamanho superior a $70 \mathrm{~mm}$ de comprimento total atingem 50\%, e de $120 \mathrm{~mm}$ atingem 100\% da primeira maturação gonadal na Baía de Paranaguá. O recrutamento ocorre durante todo o ano, indicado pelas modas de CT $=40-50$ $\mathrm{mm}$, sugere um amplo período reprodutivo. BeMvenuti (1987) caracteriza a desova desta espécie como parcelada com duração de quatro meses no estuário da Lagoa dos Patos, sendo uma das características da família Atherinopsidae. SANTos et al. (1999) encontrou um amplo período de recrutamento para esta espécie na Baía de Todos os Santos. Os resultados do presente trabalho apontam para um período ainda mais amplo e se diferenciam do sugerido por Paiva Filho \& Gianinni (1990) onde sugerem uma desova única e bem definida com recrutamento a partir de novembro, no complexo baía-estuário de São Vicente.

Atherinella brasiliensis cresce cerca de $90 \mathrm{~mm}$ durante o primeiro ano de vida, e as populações do manguezal de Guaratiba são representadas por três coortes indicadas pelo programa FISAT. O elevado valor de $\mathrm{K}(0,90)$ coincidiu com $\mathrm{o}$ encontrado por Pessanha \& Araújo (2001), sugerindo ser uma espécie de crescimento rápido. O tamanho assintótico $(\mathrm{L} \infty)$ de 168,4 mm CT foi bem superior ao de 112,6 mm CT encontrado por Pessanha \& ARAújo (2001), uma indicação da diferenciação na estrutura de tamanho da população desta espécie entre as praias arenosas da Baía de Sepetiba e o manguezal de Guaratiba. Isto sugere que o manguezal de Guaratiba pode estar apresentando melhores condições ambientais e disponibilidade de recursos quando comparados com as praias.

Os locais 2, 3 e 4 apresentaram comparativamente maiores salinidades, condutividade e oxigênio dissolvido, com esta ultima variável também sendo elevada no local 5. As maiores profundidades na zona intermediária do canal de manguezal, concentrando águas de maiores salinidade, poderiam estar associadas ao padrão espacial detectado. O oxigênio dissolvido foi o único fator a apresentar variação significativa no ciclo diuturnal. Somente a salinidade, dentre os parâmetros ambientais analisados, apresentou consistente relação positiva com a abundância de $A$. brasiliensis, tanto em número como em peso. Estas associações significativas provavelmente não implicam em relações de dependências do tipo causa-efeito, uma vez que a espécie se distribui em uma ampla variação de salinidade. Em lagoas costeiras onde apresenta elevadas densidades, a salinidade tem apresentado amplas variações como na lagoa Rodrigo de Freitas, onde a salinidade variou entre 13 e 23 (Andreata et al. 1997), e Marapendi, entre 2 e 16 (ANDREATA et al. 1990).

O uso do manguezal de Guaratiba, uma área de elevada e consistente salinidade, parece estar associado à grande capacidade destes peixes de utilizarem este tipo de ambiente altamente estruturado e de elevada disponibilidade de recursos. A estrutura de tamanho observada indica que esta espécie, representada por diferentes classes de tamanho em todos os locais e períodos do ano, completa seu ciclo de vida nesta área, com um prolongado período de recrutamento a partir de prováveis desovas nas praias arenosas próximas da ligação com mar, e o desenvolvimento das formas jovens e adultas ocorre no ambiente de manguezal. Adicionalmente a preferencia de uma espécie por habitats mais estruturados, traria a vantagem de evitar competição com formas juvenis de peixes marinhos que usam amplas áreas de praias arenosas de baías como área de recrutamento durante um determinado período do ano.

\section{AGRADECIMENTOS}

A toda a equipe de estagiários e funcionários do Laboratório de Ecologia de Peixes (UFRRJ). Ao Conselho Nacional de Desenvolvimento Científico e Tecnológico (CNPq), pelo suporte financeiro através de auxílio pesquisa (Processo: 522317/96-0) e pela bolsa de Iniciação Científica do primeiro autor (PIBIC/ UFRRJ) e bolsa de Produtividade de Pesquisa ao último autor. Ao Centro Tecnológico do Exército (CETEx), especialmente ao Capitão Reimão, e ao Campo de provas da Marambaia, especialmente ao Coronel Cardoso, por terem permitido o acesso a área do manguezal de Guaratiba. Aos funcionários do Clube Marambaia por disponibilizar suas instalações durante as coletas de campo.

\section{REFERÊNCIAS BIBLIOGRÁFICAS}

Andreata, J.V.; L.R.R. Barbiéri; A.S.C. Sebília; M.H.C. Silva; M.A. SANTOS \& R.P SANTOS. 1990. Relação dos peixes da Laguna de Marapendi, Rio de Janeiro, Brasil. Atlântica, Rio Grande, 12 (1): 5-17.

Andreata, J.V.; A.M. SaAd; L.A.F. Moraes; C.L. Soares \& A.G. MarCA. 1992. Associações, similaridade e abundância relativa dos peixes da Laguna de Jacarepaguá, Rio de Janeiro, Brasil. Boletim do Museu Nacional, Rio de Janeiro, 355: 1-25.

Andreata, J.V.; A.G. Marca; C.L. Soares \& R.S. Santos. 1997. Distribuição mensal dos peixes mais representativos da Lagoa Rodrigo de Freitas, Rio de Janeiro, Brasil. Revista Brasileira de Zoologia, Curitiba, 14 (1): 121-134.

Araújo, F.G.; A.G. Cruz-Filho; M.C. Azevedo; A.C.A. Santos \& L.A.M. Fernandes. 1997. Estrutura da comunidade de peixes jovens da margem continental da Baía de Sepetiba, RJ. Acta Biológica Leopoldensia, São Leopoldo, 19 (1): 61-83.

Bemvenuti, M.A. 1987. Abundância, distribuição e reprodução de peixes-rei (Atherinidae) na região estuarina da Lagoa dos Patos, RS, Brasil. Atlântica, Rio Grande, 9 (1): 5-32.

Bemvenuti, M.A. 1990. Hábitos alimentares de Peixes-rei (Atherinidae) na região estuarina da Lagoa dos Patos, RS, Brasil. Atlântica, Rio Grande, 12 (1): 79-102.

Blaber, S.J.M. \& T.G. Blaber. 1980. Factores affecting the distribution of juvenile estuarine and inshore fish. Journal of Fish Biology, Dunscore, 17: 143-162.

Burrows, M.T.; R.N. Gibson; L. RobB \& C.A. Commely. 1994. Temporal patterns of movement in juvenile flatfishes and their predators: underwater television observations. Journal of Experimental Marine Biology and Ecology, Sydney, 2: 215-238. 
Favaro, L.F. DE; C.G. Lopes \& H.L. Spach. 2003. Reprodução do peixe-rei, Atherinella brasiliensis (Quoy \& Gaimard) (Atheriniformes, Atherinidae), em uma planície de maré adjacente a gamboa do Baguaçu, Baía de Paranaguá, Paraná, Brasil. Revista Brasileira de Zoologia, Curitiba, 20 (3): 501-506.

Gaelzer, L.R.; G.R. Machado; O.R. Baptista \& I.R. Zalmon. 2004. Surf-zone ichthyofauna diel variaton in Arraial do Cabo, southeastern Brazil. Journal of Coastal Research, Royal Palm Beach, Special Issue, 39: 1-5.

Gayanilo-Jr, F.C. \& D. Pauly. 1997. The FAO-IClarm Stock Assessment Tools (FISAT) Reference Manual. FAO Computerized Information Series (Fisheries), Rome, 8: 1-262p.

Giannini, R.G. \& A.M. Paiva-Filho. 1995. Análise comparativa da ictiofauna da zona de arrebentação de praias arenosas do estado de São Paulo, Brasil. Boletim do Instituto Oceanográfico, São Paulo, 43 (2): 141-152.

Gomes, M.P.; M.S. CunHA, \& I.R. ZaLmon. 2003. Spatial and temporal variations of diurnal ichtthyofauna on surf-zone of São Francisco do Itabapoana beaches, Rio de Janeiro State, Brazil. Brazilian Archives of Biology and Technology, Curitiba, 46 (4): 653-664.

Hostim-Silva, M.; L. Clezar; G.C. Ribeiro \& C. Machado. 1995. Estrutura populacional de Xelomelanires brasiliensis (Quoy \& Gaimard, 1824) (Osteichthyes - Atherinidae) na Lagoa da Conceição, SC, Brasil. Arquivos de Biologia e Tecnologia, Curitiba, 38 (3): 949-960.

LaedsgaARd, P. \& C. Johnson. 2001. Why do juvenile fish utilize mangrove habitats, Australia. Journal of Experimental Marine Biology and Ecology, Australia, 257: 229-253.

Middaugh, D.P.; G.I. Scott \& J.M. Dean. 1981. Reproductive behavior of the Atlantic silverside, Menidia menidia (Pisces, Atherinidae). Environmental Biology of Fishes, Dordrecht, $6(3 / 4): 269-276$.

Middaugh, D.P. \& M.J. Hemmer. 1992. Reproductive ecology of the inland silverside, Menidia menidia, (Pisces: Atherinidae) from Blackwater Bay, Florida. Copeia, Lawrence, 1: 53-61.

Monteiro-Neto, C.; C. Blacher; A.A.S. Laurent; F.N. SnizeK; M.B. CANOZZI \& L.L.C.DE Tabajara. 1990. Estrutura de comunidade de peixes em águas rasas na região de Laguna, Santa Catarina, Brasil, Atlântica, Rio Grande, 12 (2): 43-55.

Oliveira-Neto, J.F. DE; R.S. Godefroid; G.M.N. Queiroz \& R.S. JUNIOR. 2004. Variação diuturna na captura de peixes em uma planície de maré da Baía de Paranaguá, PR. Acta Biológica Leopoldensia, São Leopoldo, 26 (1): 125-138.

Paiva-Filho, A.M. \& R.G. Giannini. 1990. Contribuição ao conhecimento da biologia do peixe-rei, Xenomelaniris brasiliensis (Quoy \& Gaimard, 1824) (Atherinidae), no complexo baíaestuário de Santos e São Vicente, Brasil. Boletim do Instituto Oceanográfico, São Paulo, 38 (1): 1-9.

Pessanha, A.L.M. \& F.G. Araújo. 2001. Recrutamento do peixerei, Atherinella brasiliensis (Guoy \& Gaimard) (Atheriniformes, Atherinopsidae), na margem continental da Baía de Sepetiba Rio de Janeiro, Brasil. Revista Brasileira de Zoologia, Curitiba, 18 (4): 1265-1274.

Pessanha, A.L.M. \& F.G. Araújo. 2003. Spatial, temporal and diel variations of fish assemblages at two sandy beaches in the Sepetiba Bay, Rio de Janeiro, Brasil. Estuarine, Coastal and Shelf Science, London, 57: 817-828.

Pessanha, A.L.M.; F.G. Araújo; M.C.C. Azevedo \& I.D. Gomes. 2000. Variações temporais e espaciais da comunidade de peixes jovens da Baía de Sepetiba, Rio de Janeiro. Revista Brasileira de Zoologia, Curitiba, 17: 251-261.

Santos, A.C.A.; F.R.C. Castullucci; C.F. Nepomuceno; E.P. Santos \& M.P. SEnA. 1999. Distribuição e recrutamento do peixe-rei Atherinella brasiliensis (Osteichthyes, Atherinidae) na margem continental oeste de Baía de Todos os Santos, Bahia, Brasil. Acta Biologica Leopoldensia, São Leopoldo, 21 (1): 107-118.

SEMA, 1998. Macroplano de gestão e saneamento ambiental da bacia da Baía de Sepetiba. Rio de Janeiro, Secretaria do Estado de Meio Ambiente do Rio de Janeiro, vol. 1.

Spach, H.L.; C. Santos \& R.S. Godefroid. 2003. Padrões temporais na assembléia de peixes na gamboa do Sucuriú, Baía de Paranaguá, Brasil. Revista Brasileira de Zoologia, Curitiba, 20 (4): 591-600.

ZAR, J.H. 1999. Biostatistical analysis. New Jersey, Prentice Hall, $4^{\text {th }}$ ed., $663 p$.

Recebido em 01.VII.2005; aceito em 02.V.2006. 